\title{
Distributed String Matching Algorithm on the $N$-cube
}

\author{
Fouzia Moussouni Christian Lavault \\ LIPN, CNRS URA 1507 \\ Université Paris-XIII, \\ Av. J.-B. Clément 93430 Villetaneuse. France \\ email: mouf@lipn.univ-paris13.fr
}

\begin{abstract}
In this paper, we present a distributed algorithm which runs on the $N$-cube and solves the string matching problem. A basic prefixsuffix matching technique is used as a building block for the construction of the algorithm. As opposed to the parallel algorithms so far designed on shared-memory PRAM models, our algorithm runs distributively on fixed hypercube networks and especially applies to long texts. Given a pattern $x$ and a text $t$ of size $n$, the occurrences of $x$ in $t$ are found in time $O(n / N+\log N)$ on the $N$-cube, including the preprocessing computation time.
\end{abstract}

\section{Introduction}

Let $t[1 . . n]$ be the text and $x[1 . . m]$ the pattern, with the two strings taken over an alphabet $\Sigma$. In the string matching problem, we want to know whether $x$ is a substring of $t$, and if so wherever $x$ matches $t$, i.e where the starting positions of all the occurrences of $x$ are in $t$.

Since the 80 's, the string matching problem was extensively studied on the Parallel RAM computation model. The first work optimal parallel string matching algorithm was given in [6], for the CRCW/PRAM. By introducing the idea of sampling in [7], Vishkin achieved an $O\left(\log ^{*} m\right)$ time (work optimal) algorithm for the problem. Galil improved this result to a constant, though with a high time overcost due to a slow preprocessing.

Recently, Gạsieniec et al. speeded up the preprocessing to $O(\log \log m)$ time, and designed a new recursive parallel divide and conquer algorithm based on the pseudo-period technique (see [2]).

On more realistic models with fixed interconnection networks and non-shared memory, the routing must be taken into account. As an example, a parallel pattern matching algorithm is considered in [1] on a mesh connected array of processors. It is also based on the pseudo-period technique and runs in time $O(\sqrt{n})$ on a $\sqrt{n} \times \sqrt{n}$ mesh computation model. Although non optimal, current algorithms on such models have a fine-granularity. One can use the same techniques with fewer number of processors. Each processor deals with $s$ successive symbols of the text by simulating $s$ virtual processors in its local memory. But it turns out to be unfeasible when having quite a long text per processor. More exactly, on a parallel computer with $N$ fixed processors and a reasonably big ratio $\frac{n}{N}$, it 
is more efficient to use sequential computations in parallel and perform limited communication. Without loss of generality we may assume that $N$ divides $n$ (otherwise, the text $t$ may be padded on the right with extra symbols). Each processor deals with a text segment $T_{i}$ of size $\left|T_{i}\right|=s=\left\lceil\frac{n}{m}\right\rceil \geq m$. Some of the $N$-cube's links connect the end of each segment $T_{i}$ to the beginning of the next segment $T_{i+1}$. By contrast with the classical string matching problem, there exist pattern occurrences that span two consecutive segments of the text. We use a prefix-suffix matching technique as a basic building block of the algorithm for finding all overlapping occurrences caused by the text segmentation. We show that the overall string matching problem can be solved in time $O(s)$, in constant space, and linear total work on a MIMD hypercube with no more than $N$ processors.

\section{The parallel computation model}

Many computation models may be thought of to deal with string processing, e.g. the hypercube network was chosen herein for its versatility. The $d$-dimensional hypercube $Q_{d}$ has $N=2^{d}$ processors which communicate by exchanging messages through its $d \cdot 2^{d-1}$ links. An important property is that the hypercube can simulate many known networks $(O(N)$-node arrays, rings, meshes, binary trees, etc.). This way of looking at the hypercube makes it possible to implement simple parallel algorithms on any such networks while using the attractive properties of $Q_{d}$. To have a good task mapping, string matching algorithms use general simulations on lines, rings (e.g, pipelining or simple linear string matching algorithms), and similarly on meshes with a snake-like ordering of the pattern.

\section{$3 \quad N$-cube String Matching with Long Texts}

The text $t$ is first broken into non-overlapping $s$-blocks and then distributed over the PEs such that each $P E_{i}$ contains subtext $T_{i}=t_{s i+1} \ldots t_{s(i+1)}$. We say that position $j$ in the text is at position $j^{\prime}$ in $T_{i}$ whenever $j=i \cdot s+j$, where $s=\left\lceil\frac{n}{N}\right\rceil$. By using a spanning tree embedding technique for broadcasting $O(m)$ items to all $P E \mathrm{~s}$, the pattern is broadcasted to all other $P E \mathrm{~s}$. The overall time needed for this initialization phase is bounded by $O(m+\log N)$. The allocation scheme places adjacent segments of the text on adjacent processors. Thus, what we want to do is to find all the occurrences of the pattern across multiple segments by serial computations in each processor.

The main difficulty is that the pattern may occur in some subtext $T_{i}$ as a substring overlapping two adjacent segments, viz. either $T_{i}$ and $T_{i+1}$, or $T_{i}$ and $T_{i-1}$, caused by the text segmentation.

To avoid text block transfers, our algorithm operates according to whether the pattern has some periods or none. The possible periodicity of the pattern $x$ is the key part of the algorithm. 
We search for occurrences that are within the subtexts by running the fastest sequential string matching algorithm on each processor. Now, to find occurrences in the overlapping endpoints of a segment $T_{i}$, we need to compute some prefixsuffix information. Recall that a subword $u$ of the pattern is said to be a prefix of $x$ if $x=u z$. Similarly, a subword $v$ of the pattern is a suffix of $x$ if $x=z v$.

To find occurrences that occur in two segments $T_{i}$ and $T_{i+1}$, each processor $P E_{i}$ sends to $P E_{i+1}$ one constant size message carrying the prefix length $|u|$ (if any). Because of the periodicity property, we also consider the longest suffix $v$ located at the beginning of $T_{i+1}$. Upon receipt of the prefix length value, the processor $P E_{i+1}$ checks which of the following property holds:

1. $|u|+|v|=m$ and the pattern $x$ is strictly nonperiodic (i.e., without periodic prefixes and suffixes).

2. $|u|+|v| \equiv m \quad(\bmod p)$ and $x$ is periodic with shortest period $p$.

3. $p_{x} \equiv|u|+|v|-m(\bmod p)$ and $x$ is periodic with shortest period $p, p_{x}$ being a greater period of $x$ and nonmultiple of $p$.

4. $|u|+|v| \equiv m \quad(\bmod p p($ resp. $s p))$ and $x$ is partially periodic. Which means that $x$ is not periodic while some prefix (resp. suffix) is periodic with period $p p$ (resp. $s p$ ).

In whichever case, there is at least one pattern occurrence across the sections $T_{i}$ and $T_{i+1}$. The corresponding position in the text $t$ clearly depends on which of the above properties holds. More details can be found in [5].

Note that only one constant size message carrying the prefix size $|u|$ is sent to processor $P E_{i+1}$. An additional linear time (in the pattern size) is used for searching the words $u$ and $v$, which is negligible with respect to the global communication time. Finally, an upper bound on local time complexity is $O(s)$ since we run a serial string matching algorithm to match both the word $x$, the prefix $u$ and the suffix $v$. The space complexity related to this string matching algorithm is either linear in the pattern size (e.g using KMP) or a constant. Since $m=O\left(\frac{n}{N}\right)$, this provides a sketchproof of the following proposition:

Proposition Taking the preprocessing into account, the time complexity of the above $N$-cube string matching algorithm is $O\left(\frac{n}{N}+\log (N)\right)$.

\section{Concluding Remarks}

We proposed a new flexible distributed algorithm which solves the string matching problem on a fixed $N$-cube with long texts stored on processors. Compared to classical parallel string matching algorithms, the text has a broken structure caused by the distribution of computations in non-shared memory models. So, we consider that pattern occurrences may overlap adjacent segments. 
For finding all pattern occurrences, we presented a more involved linear time algorithm, with pattern periods assumptions. Note that, a kind of trade-off between the size of the hypercube and the time complexity may be achieved to give the first optimal algorithm with the smallest possible size of the cube.

Open questions. Design a more general algorithm to search for long texts as well as small texts allocated to processors. Kim and Park address a similar problem in [4] in the case of an hypertext which is also a nonlinear structure of a text.

Extend the relevant prefix-suffix technique (mainly used to deal with overlapping occurrences), to sequential and incremental string matching problems (see [3]). All pattern occurrences may be derived by matching maximal prefixes and suffixes on independent blocks of the text. Using this technique, a linear algorithm in the number of comparisons is expected.

\section{References}

1. B.S. Chlebus and L. Gassieniec. Optimal pattern matching on meshes. In 11th Ann. Symp. on Theoretical Aspects of Computer Science, pages 213-224, 1994.

2. A. Czumaj, Z. Galil, L. Gạsieniec, K. Park, and W. Plandowski. Work-time optimal parallel algorithms for strings problems. Manuscript, 1994.

3. Z.M. Kedem, G.M. Landau, and K.V. Palem. Optimal parallel suffix-prefix matching algorithm and applications. In ACM Symposium on Parallel Algorithms and Architectures, pages 388-398, 1989.

4. D.K. Kim and K. Park. String matching in hypertext. In 6th Annuel Symp. on Combinatorial Pattern Matching, Lecture Notes in Computer Science, pages 318 329, 1995.

5. C. Lavault and F. Moussouni. N-cube string matching algorithm with long texts. In The Proceeding of CCS'95, Combinatorics and Computer Science, To appear in Lecture Notes in Computer Science, 1995.

6. U. Vishkin. Optimal parallel pattern matching in strings. Information and Control, 67:91-113, 1985.

7. U. Vishkin. Deterministic sampling - a new technique for fast pattern matching. SIAM J.Comput, 20:22 40, 1991. 